\title{
MODULES WHICH ARE INVARIANT UNDER MONOMORPHISMS OF THEIR INJECTIVE HULLS
}

\author{
A. ALAHMADI, N. ER ${ }^{\curvearrowleft}$ and S. K. JAIN \\ (Received 5 December 2003; revised 30 November 2004)
}

Communicated by J. Du

\begin{abstract}
In this paper certain injectivity conditions in terms of extensions of monomorphisms are considered. In particular, it is proved that a ring $R$ is a quasi-Frobenius ring if and only if every monomorphism from any essential right ideal of $R$ into $R_{R}^{(\mathbb{N})}$ can be extended to $R_{R}$. Also, known results on pseudo-injective modules are extended. Dinh raised the question if a pseudo-injective CS module is quasi-injective. The following results are obtained: $M$ is quasi-injective if and only if $M$ is pseudo-injective and $M^{2}$ is CS. Furthermore, if $M$ is a direct sum of uniform modules, then $M$ is quasi-injective if and only if $M$ is pseudo-injective. As a consequence of this it is shown that over a right Noetherian ring $R$, quasi-injective modules are precisely pseudo-injective CS modules.
\end{abstract}

2000 Mathematics subject classification: primary 16D50, 16D70.

\section{Introduction}

Throughout the paper rings are associative with identity and modules are unitary (right) modules. Let $M$ and $N$ be two right $R$-modules over a ring $R$. $M$ is called (pseudo-) $N$ injective if, for any submodule $A$ of $N$, every homomorphism (monomorphism) in $\operatorname{Hom}_{R}(A, M)$ can be extended to an element of $\operatorname{Hom}_{R}(N, M) . M$ is called quasiinjective (pseudo-injective) if it is (pseudo-) $M$-injective. $M$ and $N$ are called relatively injective if $M$ is $N$-injective and $N$ is $M$-injective. A submodule $K$ of $M$ is said to be a complement in $M$ of a submodule $B$ if $K$ is a maximal submodule among those that have zero intersection with $B$. Complement submodules of $M$ coincide with the submodules of $M$ which do not have any proper essential extension in $M$. Also, if $A$ is a complement in $M$ and $B$ is a complement in $A$, then $B$ is a complement in $M$.

(C) 2005 Australian Mathematical Society $1446-7887 / 05 \$ A 2.00+0.00$ 
A CS module is one in which complement submodules are direct summands. $M$ is called a continuous module if it is a CS module and submodules of $M$ isomorphic to direct summands of $M$ are again direct summands. If $M$ is continuous and $A$ and $B$ are two direct summands of $M$ with $A \cap B=0$, then $A \oplus B$ is also a direct summand of $M$. The hierarchy is as follows:

$$
\text { Injective } \Longrightarrow \text { quasi-injective } \Longrightarrow \text { continuous } \Longrightarrow \mathrm{CS} \text {. }
$$

For other properties of complements and CS/continuous modules and the proofs of the above mentioned properties, the reader is referred to [3] and [10].

In this paper, a weaker form of pseudo- $N$-injectivity is considered, and it is proved, in particular, that a ring $R$ is quasi-Frobenius if and only if monomorphisms from essential right ideals of $R$ into $R^{(\mathbb{N})}$ can be extended to $R_{R}$. Also it is shown that a module $M$ is invariant under monomorphisms of its injective hull if and only if every monomorphism from any essential submodule of $M$ can be extended to $M$. This extension property is used to characterize when semi-prime/right nonsingular rings are SI (see [6]).

Pseudo-injectivity has been studied by several authors such as Dinh, Jain, Singh, Teply, Tuganbaev and others (see [2, 8, 9, 13-15]). It was first introduced by Jain and Singh [8]. Teply [14] constructed examples of pseudo-injective modules which are not quasi-injective. In [2] Dinh raised the question if a pseudo-injective CS module is quasi-injective. He stated in [2] that the answer is affirmative if we assume further that $M$ is nonsingular. In this paper we prove the following: $M$ is quasi-injective if and only if $M$ is pseudo-injective and $M^{2}$ is CS. Every uniform pseudo-injective module is quasi-injective. Consequently, over a right Noetherian ring $R$, quasi-injective modules are precisely pseudo-injective CS modules.

\section{Essentially pseudo- $N$-injectivity}

In this section we consider a weaker form of pseudo- $N$-injectivity.

DEFINITION 2.1. Let $M$ and $N$ be two modules. $M$ is said to be essentially pseudo$N$-injective if for any essential submodule $A$ of $N$, any monomorphism $f: A \rightarrow M$ can be extended to some $g \in \operatorname{Hom}(N, M) . M$ is called essentially pseudo-injective if $M$ is essentially pseudo- $M$-injective.

Obviously any pseudo- $N$-injective module is essentially pseudo- $N$-injective, but the converse is not true in general.

EXAMPLE 1 . Let $p$ be a prime. The $\mathbb{Z}$-module $\mathbb{Z} / p^{2} \mathbb{Z}$ is not pseudo-( $\left.\mathbb{Z} \oplus \mathbb{Z} / p^{3} \mathbb{Z}\right)$ injective since the obvious isomorphism $\iota: p \mathbb{Z} / p^{3} \mathbb{Z} \rightarrow \mathbb{Z} / p^{2} \mathbb{Z}$ can not be extended 
to any element of $\operatorname{Hom}\left(\mathbb{Z} \oplus \mathbb{Z} / p^{3} \mathbb{Z}, \mathbb{Z} / p^{2} \mathbb{Z}\right)$, but it is essentially pseudo-( $\mathbb{Z} \oplus \mathbb{Z} / p^{3} \mathbb{Z}$ )injective.

The following proposition provides a characterization of essentially pseudo- $N$ injectivity.

Proposition 2.2. Let $M$ and $N$ be two modules and $X=M \oplus N$. The following conditions are equivalent:

(i) $M$ is essentially pseudo- $N$-injective.

(ii) For any complement $K$ in $X$ of $M$ with $K \cap N=0, M \oplus K=X$.

ProOF. (i) $\Rightarrow$ (ii) Let $K$ be a complement in $X$ of $M$ with $K \cap N=0$, and $\pi_{M}: M \oplus N \rightarrow M$ and $\pi_{N}: M \oplus N \rightarrow N$ be the obvious projections. Note that $M \oplus K=M \oplus \pi_{N}(K)$ so that $\pi_{N}(K)$ is essential in $N$.

Now define $\theta: \pi_{N}(K) \rightarrow \pi_{M}(K)$ as follows: For $k \in K$ with $k=m+n$ ( $m \in M, n \in N$ ), $\theta(n)=m$. Then $\theta$ is a monomorphism by the $K \cap N=0$ assumption. Hence $\theta$ can be extended to some $g: N \rightarrow M$, since $M$ is essentially pseudo- $N$-injective. Now let $T=\{n+g(n): n \in N\}$. It is easy to see that $M \oplus T=X$. Also, $T$ contains $K$ essentially by modularity. Since $K$ is a complement, this implies $T=K$. Now the conclusion follows.

(ii) $\Rightarrow$ (i) Assume (ii). Let $A$ be an essential submodule of $N$ and $f: A \rightarrow M$ be a monomorphism. Let $H=\{a-f(a): a \in A\}$. Obviously, $H \cap N=0$. Also note that $M \oplus H=M \oplus \pi_{N}(H)=M \oplus A$, which is essential in $X$. Let $K$ be a complement in $X$ of $M$ containing $H$. By the previous argument and modularity $H$ is essential in $K$, so that $K \cap N=0$. By assumption we have $M \oplus K=X$. Now let $\phi: M \oplus K \rightarrow M$ be the obvious projection. Then the restriction $\phi_{\mid N}$ is the desired extension of $f$. The proof is now complete.

PROPOSITION 2.3. If $M$ is essentially pseudo- $N$-injective, every direct summand of $M$ is essentially pseudo- $N$-injective.

Proof. Let $X=M \oplus N$ and assume $M=M_{0} \oplus A$. Let $K$ be a complement in $M_{0} \oplus N$ of $M_{0}$ with $K \cap N=0$. Then $M \oplus K$ is essential in $X$. Since $K$ is a complement submodule, the preceding argument implies that $K$ is also a complement in $X$ of $M$. Now by Proposition 2.2 $M \oplus K=X$. Then $M_{0} \oplus K=M_{0} \oplus N$, which yields the conclusion again by Proposition 2.2.

The next example shows that essentially pseudo- $\mathrm{N}$-injectivity is not inherited by direct sums. 
EXAMPLE 2. Let $F$ be a field and

$$
R=\left(\begin{array}{cc}
F & F \oplus F \\
0 & F
\end{array}\right)
$$

Consider the $R$-modules

$$
N=\left(\begin{array}{cc}
F & F \oplus F \\
0 & 0
\end{array}\right), \quad S_{1}=\left(\begin{array}{cc}
0 & 0 \oplus F \\
0 & 0
\end{array}\right), \quad S_{2}=\left(\begin{array}{cc}
0 & F \oplus 0 \\
0 & 0
\end{array}\right) .
$$

Then $S_{1}$ and $S_{2}$ are both essentially pseudo- $N$-injective. But since the identity map of $S_{1} \oplus S_{2}$ obviously can not be extended to an element of $\operatorname{Hom}\left(N, S_{1} \oplus S_{2}\right), S_{1} \oplus S_{2}$ is not essentially pseudo- $N$-injective.

PROPOSITION 2.4. Let $M$ and $N$ be two modules. Then the following conditions are equivalent:

(i) $M$ is $N$-injective.

(ii) $M$ is essentially pseudo- $N / L$-injective for every submodule $L$ of $N$.

ProOf. (i) $\Rightarrow$ (ii) follows from [10, Proposition 1.3].

(ii) $\Rightarrow$ (i) Assume $M$ is essentially pseudo- $N / L$-injective for every submodule $L$ of $N$. Let $X=M \oplus N, A \subseteq X$ with $A \cap M=0$ and $K$ be a complement in $X$ of $M$ containing $A$. Also let $T=K \cap N$. Since $(M \oplus K) / K$ is essential in $X / K$, then $(M \oplus K) / T$ is essential in $X / T$, and $K / T \cap N / T=0$. Thus it is easy to see that $K / T$ is a complement in $X / T$ of $(M \oplus T) / T$. Now by assumption and Proposition 2.2 we have $(M \oplus T) / T \oplus K / T=X / T$. Hence $M \oplus K=X$. Then by [3, Lemma 7.5] $M$ is $N$-injective.

COROLLARY 2.5. $M$ is injective if and only if $M$ is essentially pseudo- $N$-injective for any cyclic module $N$.

COROLLARY 2.6. A nonsingular module $M$ is injective if and only if it is essentially pseudo- $N$-injective for any nonsingular cyclic module $N$.

Proof. Let $A$ be any cyclic module and $B$ be an essential submodule of $A$. Let $f: B \rightarrow M$ be a monomorphism. Then $A$ is obviously nonsingular, so that $f$ can be extended to some $g: A \rightarrow M$ by assumption. Now the result follows by Corollary 2.5 .

The following result generalizes [2, Theorem 2.2] and [9, Theorem 1].

THEOREM 2.7. If $M \oplus N$ is essentially pseudo- $N$-injective, then $M$ is $N$-injective. 
Proof. Call $X=M \oplus N$. Let $A$ and $K$ be as in the proof of Proposition 2.4. Let $\pi: M \oplus N \rightarrow N$ be the obvious projection. Then $M \oplus K=M \oplus \pi(K)$ and thus $\pi(K)$ essential in $N$. Note that $K \cong \pi(K)$. Pick any isomorphism $f: \pi(K) \rightarrow K$. By assumption $f$ can be extended to some monomorphism $g: N \rightarrow X$. Then $g(\pi(K))=K$ is essential in $g(N)$. But since $K$ is a complement in $X$, we must have $K=g(N)$, whence $\pi(K)=N$. Thus $M \oplus K=X$. Now the result follows by [3, Lemma 7.5].

COROLLARY 2.8. $M$ is quasi-injective if and only if $M^{2}$ is essentially pseudo-Minjective.

Osofsky proved in [12] that a ring $R$ is semisimple Artinian if and only if every cyclic right (left) $R$-module is injective.

COROLLARY 2.9. A ring $R$ is semisimple Artinian if and only if every countably generated right $R$-module is essentially pseudo-injective.

Proof. Let $M$ be a cyclic right $R$-module. Then $(M \oplus R)^{(\mathbb{N})} \cong(M \oplus R)^{(\mathbb{N})} \oplus$ $(M \oplus R)^{(\mathbb{N})}$, which is countably generated, whence essentially pseudo-injective. Thus $\left(M \oplus R^{(\mathbb{N})}\right)^{2}$ is essentially pseudo- $\left(M \oplus R^{(\mathbb{N})}\right)$-injective. Then by Theorem 2.7, $\left(M \oplus R^{(\mathbb{N})}\right)$ is quasi-injective, whence $R_{R}$-injective. Therefore $M$ is injective. Now the conclusion follows by Osofsky's theorem.

COROLlARY 2.10 ([2, Theorem 2.2]). If $M \oplus N$ is pseudo-injective, then $M$ and $N$ are relatively injective.

In what follows $E(M)$ stands for the injective hull of $M$ and we will consider $M$ as a submodule of $E(M)$. We will also use the notation $E_{N}(M)$ for the submodule of $E(M)$ generated by all the isomorphic copies of $N$. Note that $E_{N}(M)$ is invariant under monomorphisms of $\operatorname{End}(E(M))$ and that $E_{R_{R}}(M)$ contains all elements of $M$ with zero right annihilator in $R$.

PROPOSITION 2.11. $M$ is essentially pseudo- $N$-injective if and only if $E_{N}(M) \subseteq M$.

ProOF. Assume $E_{N}(M) \subseteq M$ and let $B$ be an essential submodule of $N$, and $f: B \rightarrow M$ be a monomorphism. There exists some monomorphism $g: N \rightarrow E(M)$ such that $g_{1 B}=f$. By assumption $g(N) \subseteq M$. Thus $g$ is the desired extension of $f$, whence $M$ is essentially pseudo- $N$-injective.

Conversely assume that $M$ is essentially pseudo- $N$-injective. We will use the same argument as in [10, Lemma 1.13]: Let $h: N \rightarrow E(M)$ be a monomorphism. Let $A=h^{-1}(M)$. Then $A$ is essential in $N$. Thus, by assumption, the restriction $h_{\mid A}$ extends to some $\theta: N \rightarrow M$. Now assume $h(n) \neq \theta(n)$ for some $n \in N$. Then 
$x=h(n)-\theta(n) \neq 0$. Since $M$ is essential in $E(M)$, there exists some $r \in R$ such that $0 \neq x r=h(n r)-\theta(n r) \in M$. But then $h(n r) \in M$ so that $n r \in A$. This is a contradiction since $\theta_{\mid A}=h_{\mid A}$. Now the conclusion follows.

COROLLARY 2.12. $M$ is essentially pseudo-injective if and only if it is invariant under monomorphisms in $\operatorname{End}(E(M))$.

COROLLARY 2.13. Let $\left\{A_{i}\right\}$ be a family of submodules of a module $N, B=\Sigma A_{i}$ and assume $M$ is essentially pseudo- $A_{i}$-injective for each $i$. Then $M$ is essentially pseudo-B-injective.

Proof. Let $f: B \rightarrow E(M)$ be a monomorphism. Then $f(B)=\Sigma f\left(A_{i}\right)$. By assumption and Proposition 2.11, $f(B)$ is contained in $M$. Now the conclusion follows again by Proposition 2.11 .

The converse of the Corollary 2.13 does not hold in general.

EXAMPLE 3. Let $p$ be a prime. It is easy to see that the $\mathbb{Z}$-module $\mathbb{Z} / p^{2} \mathbb{Z}$ is not essentially pseudo- $\mathbb{Z} / p^{3} \mathbb{Z}$-injective, but it is trivially essentially pseudo-( $\mathbb{Z} \oplus \mathbb{Z} / p^{3} \mathbb{Z}$ )injective.

COROLlaRY 2.14. Let $E$ be an injective module and $A$ be any submodule of $E$. Then $X=\Sigma\{C \mid C \leq E, C \cong A\}$ is essentially pseudo-injective.

PROOF. First note that $E(X)$ is a summand of $E$. As in the proof of Corollary 2.13, for any monomorphism $f: X \rightarrow E(X), f(X)$ is contained in $X$. The conclusion follows by Proposition 2.11.

Goodearl defined a right SI-ring to be one over which every singular right module is injective ([6]). Such rings are precisely right nonsingular rings over which singular right modules are semi-simple (see [3]).

THEOREM 2.15. Let $R$ be a ring which is either right nonsingular or semi-prime. The following conditions are equivalent:

(i) $R$ is a right SI-ring.

(ii) Any two cyclic singular right $R$-modules are relatively essentially pseudoinjective.

(iii) For any two cyclic singular right $R$-modules $B$ and $C, E_{B}(C) \subseteq C$.

ProOF. (i) $\Rightarrow$ (ii) Trivial.

(ii) $\Leftrightarrow$ (iii) The statement follows from Proposition 2.11. 
(ii) $\Rightarrow$ (i) Assume (ii). Then cyclic singular right $R$-modules are relatively injective by Proposition 2.4. So if $C$ and $M$ are singular right $R$-modules and $C$ is cyclic, then $C$ is $M$-injective by the above argument and [10, Proposition 1.4]. This implies, by [3, Corollary 7.14], that all singular right $R$-modules are semi-simple.

Now, if $R$ is right nonsingular, the conclusion immediately follows by the preceding remark and the above argument. Else, assume that $R$ is semi-prime. Since singular modules are semi-simple, $Z\left(R_{R}\right)^{2}=0$, whence $Z\left(R_{R}\right)=0$. Now the conclusion follows by the above argument.

\section{Pseudo-injectivity}

Proposition 3.1 ([16, Corollary 2.9]). Let $M$ and $N$ be two modules and $X=$ $M \oplus N$. The following conditions are equivalent:

(i) $M$ is pseudo- $N$-injective.

(ii) For any submodule $A$ of $X$ with $A \cap M=A \cap N=0$, there exists a submodule $T$ of $X$ containing $A$ with $M \oplus T=X$.

ProOF. (i) $\Rightarrow$ (ii) Assume (i) and let $A$ satisfy the assumptions of (ii). Also let $\pi_{M}$ and $\pi_{N}$ be as in the Proposition 2.2, and define $\theta: \pi_{N}(A) \rightarrow \pi_{M}(A)$ as follows: $\theta\left(\pi_{N}(a)\right)=\pi_{M}(a)$, for $a \in A$. Then, by assumption, $\theta$ extends to some $g \in \operatorname{Hom}(N, M)$. Let $T=\{n+\theta(n) \mid n \in N\}$. Then we have $M \oplus T=X$ and $A \subseteq T$, as required.

(ii) $\Rightarrow$ (i) Assume (ii). Let $B$ be a submodule of $N$ and $f: B \rightarrow M$ be a monomorphism. Call $A=\{b-f(b) \mid b \in B\}$. Then $A \cap M=A \cap N=0$. Now, by assumption, there exists a submodule $T$ of $X$ containing $A$ with $M \oplus T=X$. Let $\pi: M \oplus T \rightarrow M$ be the obvious projection. Then the restriction $\pi_{\mid N}$ is the desired extension of $f$.

Jain and Singh proved in [8, Theorem 3.7] that for a nonsingular module $M$ with finite uniform dimension, the following conditions are equivalent: (i) $M$ is pseudo-injective; (ii) $M$ is invariant under any monomorphism (isomorphism in the terminology of [8]) of $\operatorname{End}(E(M))$ (that is, $M$ is essentially pseudo-injective by Corollary 2.12). The following result extends it to any module with finite uniform dimension.

THEOREM 3.2. Let $M$ be a module with finite uniform dimension. Assume that for any two essential submodules $D$ and $E$ of $M$, every isomorphism $h: D \rightarrow E$ can be extended to some $g \in \operatorname{End}(M)$. Then every monomorphism from any submodule of $M$ into $M$ can be extended to a monomorphism of $M$. 
In particular, a module with finite uniform dimension is pseudo-injective if and only if it is essentially pseudo-injective.

ProOF. Let $M$ be as in the former assumption, $A$ be a submodule of $M$, and $f: A \rightarrow M$ be a monomorphism. Call $B=f(A)$. Pick, by Zorn's Lemma, two submodules $A^{\prime}$ and $B^{\prime}$ of $M$ such that $A \oplus A^{\prime}$ and $B \oplus B^{\prime}$ are essential in $M$. Now, $E(M)=E(A) \oplus E\left(A^{\prime}\right)=E(B) \oplus E\left(B^{\prime}\right)$ and $E(A) \cong E(B)$. Then by [10, Theorem 1.29] and since $M$ has finite uniform dimension, we have $E\left(A^{\prime}\right) \cong E\left(B^{\prime}\right)$. Thus $A^{\prime}$ and $B^{\prime}$ have isomorphic essential submodules $U \subseteq A^{\prime}$ and $V \subseteq B^{\prime}$. Then $A \oplus U$ and $B \oplus V$ are essential submodules of $M$. And since $U$ and $V$ are isomorphic to each other, there exists an isomorphism $\theta: A \oplus U \rightarrow B \oplus V$ such that $\theta_{\mid A}=f$. By assumption $\theta$ extends to some monomorphism $g \in \operatorname{End}(M)$. Obviously, $g_{\mid A}=f$. Therefore, the conclusion follows.

Note that, in [1, Theorem 2.1], Alamelu gives a proof that $M$ is pseudo-injective if and only if $M$ is invariant under monomorphisms of $\operatorname{End}(E(M))$, where $M$ is an arbitrary module over a commutative ring (here the commutativity assumption is irrelevant to the proof). However, the proof is incorrect. In summary, the proof states that for a module $M$ which is invariant under monomorphisms of its injective hull, and for any monomorphism $f: N \rightarrow M$ where $N$ is a submodule of $M, f$ can be extended to a monomorphism $f^{\prime \prime}: E(M) \rightarrow E(M)$. This is not correct as the following example shows: Let $M$ be any directly infinite injective module with $M=N \oplus B$, where $M \cong N$ and $B$ is nonzero. Also let $f: N \rightarrow M$ be any isomorphism. Obviously, $f$ cannot be extended to a monomorphism in $\operatorname{End}(E(M)$ ).

In [4] and [5] Er studied the modules in which isomorphic copies of complements are again complements. These are called SICC-modules in [5]. The following result was proved in [8] for nonsingular modules, but the proof works for an arbitrary pseudo-injective module as well.

LEMMA 3.3 ([8, Lemma 3.1]). If $M$ is pseudo-injective, then submodules of $M$ isomorphic to complements in $M$ are again complements.

Proof. Let $K$ be a complement in $M$ and $A$ be a submodule of $M$ with an isomorphism $f: A \rightarrow K$. Then $f$ extends to some $g \in \operatorname{End}(M)$ by assumption. Pick, by Zorn's Lemma, a complement $A^{\prime}$ in $M$ essentially containing $A$. Then the restriction $g_{\mid A^{\prime}}$ is obviously a monomorphism. Hence $K=g(A)$ is essential in $g\left(A^{\prime}\right)$. Since $K$ is a complement this implies $K=g\left(A^{\prime}\right)$, whence $A=A^{\prime}$. The conclusion follows.

REMARK. Modules in which submodules isomorphic to complements are complements always decompose into relatively injective summands by [5, Lemma 4$]$. So 
Corollary 2.10 also follows from that result and Lemma 3.3. It is proved in [2, Corollary 2.8] that a pseudo-injective CS module is continuous. This result also follows from Lemma 3.3 and the definition of CS.

Dinh [2] raised the question whether a CS module $M$ which is pseudo-injective is quasi-injective, and stated in [2] that the answer is affirmative when $M$ is furthermore nonsingular. Now we present some partial answers to Dinh's question.

THEOREM 3.4. $M$ is quasi-injective if and only if $M$ is pseudo-injective and $M^{2}$ is $C S$.

Proof. Assume $M$ is pseudo-injective and $M^{2}$ is CS. Let $M_{1}$ and $M_{2}$ be two isomorphic copies of $M$ and $X=M_{1} \oplus M_{2}$. Note that $M$ is continuous by the preceding remark.

First let $A$ be any complement in $X$ with $A \cap M_{1}=0$ and $A \cap M_{2}$ essential in $A$. There exist submodules $V$ and $V^{\prime}$ of $M_{2}$ such that $V \oplus V^{\prime}=M_{2}$ and $V$ contains $A \cap M_{2}$ essentially. Also since $M^{2}$ is CS by assumption, we have $A \oplus A^{\prime}=X$ for some submodule $A^{\prime}$ of $X$. Since $V$ is a direct summand of a continuous module, $V$ is continuous (see [10]), whence it has exchange property by [10, Theorem 3.4]. Since $V \cap A$ is essential in $A$, we have $V \cap A^{\prime}=0$. Thus we must have $V \oplus A^{\prime}=X$. Hence $A$ is isomorphic to a summand, namely $V$ of $M_{2}$.

Now let $C$ be a submodule of $X$ such that $C \cap M_{1}=0$ and pick, by Zorn's Lemma, a complement $K$ in $X$ of $M_{1}$ containing $C$. Again by Zorn's Lemma, choose a complement $K_{1}$ in $K$ of $K \cap M_{2}$ and a complement $K_{2}$ in $K$ of $K_{1}$ containing $K \cap M_{2}$. Note that $K \cap M_{2}$ is essential in $K_{2}$ and that $K_{1}$ and $K_{2}$ are complements in $X$ by $[3,1.10]$. By Proposition 3.1 there exists some submodule $T$ of $X$ containing $K_{1}$ with $M_{1} \oplus T=X$. Then $T \cong M$ and $K_{1}$ is a complement in $T$, whence $K_{1}$ is isomorphic to a complement in $M_{2}$. Also by the preceding paragraph $K_{2}$ is isomorphic to a complement of $M_{2}$ too. Now consider the usual projection $\pi: M_{1} \oplus M_{2} \rightarrow M_{2}$. We have $M_{1} \oplus\left(K_{1} \oplus K_{2}\right)=M_{1} \oplus\left(\pi\left(K_{1}\right) \oplus \pi\left(K_{2}\right)\right)$, where $\pi\left(K_{i}\right) \cong K_{i}$. Hence by continuity of $M_{2}$ and the above argument, $\pi\left(K_{1}\right) \oplus \pi\left(K_{2}\right)$ is a summand of $M_{2}$. Now, since $K$ is a complement of $M_{1}, M_{1} \oplus K=M_{1} \oplus \pi(K)$ is essential in $X$. Then $\pi(K)$ is essential in $M_{2}$. Also, by choice of $K_{i}, K_{1} \oplus K_{2}$ is essential in $K$. Then $\pi\left(K_{1}\right) \oplus \pi\left(K_{2}\right)$ is essential in $\pi(K)$, hence in $M_{2}$. This implies that $M_{2}=\pi\left(K_{1}\right) \oplus \pi\left(K_{2}\right)=\pi(K)$. Thus $M_{1} \oplus K=X$. Now it follows by [3, Lemma 7.5] that $M_{1}$ is $M_{2}$-injective. The proof is now complete.

The following is a key result.

LEMMA 3.5. Let $M=\bigoplus_{i \in I} M_{i}$ be a direct sum of uniform modules $M_{i} . \quad M$ is quasi-injective if and only if it is pseudo-injective. In particular, any uniform pseudoinjective module is quasi-injective. 
ProOF. First let $M$ be a uniform pseudo-injective module. Let $A$ be a submodule of $M$ and $f: A \rightarrow M$ be a nonzero homomorphism. If $\operatorname{Ker}(f)=0$, then $f$ can be extended to an element of $\operatorname{End}(M)$ by assumption. So assume $\operatorname{Ker}(f) \neq 0$. Let $\delta=i_{A}-f$, where $i_{A}: A \rightarrow M$ is the inclusion map. Since $\operatorname{Ker}(f) \neq 0$ and $M$ is uniform, $\operatorname{Ker}(\delta)=0$. Then by pseudo-injectivity assumption $\delta$ can be extended to some $g \in \operatorname{End}(M)$. Now $1-g$ is obviously an extension of $f$. Thus $M$ is quasi-injective.

Now let $M=\bigoplus_{i \in I} M_{i}$ be a direct sum of uniform modules $M_{i}$ and assume that $M$ is pseudo-injective. Then, by Corollary $2.10, M(I-i)$ is $M_{i}$-injective for all $i \in I$. Now by the preceding paragraph and since direct summands of pseudoinjectives are obviously pseudo-injective, each $M_{i}$ is quasi-injective. Therefore $M$ is quasi-injective.

THEOREM 3.6. Over a right Noetherian ring $R$, a right $R$-module $M$ is quasiinjective if and only if $M$ is a pseudo-injective CS-module.

PROOF. Let $M$ be a pseudo-injective CS module. Then $M$ is a direct sum of uniform submodules by [11]. Now the result follows by Lemma 3.5.

Before proving the next result, note that $R$ is called a right countably $\Sigma$-CS ring if $R_{R}^{(\mathrm{N})}$ is a CS module.

THEOREM 3.7. The following conditions are equivalent for a ring $R$ :

(i) $R$ is a quasi-Frobenius ring.

(ii) Every projective right $R$-module is essentially pseudo- $R_{R}$-injective.

(iii) $R_{R}^{(\mathbb{N})}$ is essentially pseudo- $R_{R}$-injective.

(iv) $R$ is a right countably $\Sigma$-CS ring with finite uniform dimension and $R_{R}$ is essentially pseudo-injective.

Proof. The implications (i) $\Rightarrow$ (ii) and (ii) $\Rightarrow$ (iii) are obvious, and (i) $\Rightarrow$ (iv) follows from the fact that every injective module is $\mathrm{CS}$, and (iii) $\Rightarrow$ (i) follows by Theorem 2.7 .

(iv) $\Rightarrow$ (i) Since $R_{R}$ has finite uniform dimension, then $R_{R}$ is pseudo-injective by Theorem 3.2. Then by Theorem $3.4 R$ is a right self-injective ring with finite uniform dimension. Hence $R$ is a semiperfect right countably $\Sigma$-CS ring. This implies by [7] that $R$ is Artinian. Now the conclusion follows.

The following results were proved in [5, Theorem 2, Corollary 4, Theorem 3, Theorem 4] for modules in which submodules isomorphic to complements are complements. Each pseudo-injective module satisfies this property by Lemma 3.3, whence we have the following corollaries. 
COROLlaRY 3.8. Any decomposition of a pseudo-injective module into indecomposable submodules complements summands.

COROLLARY 3.9. An essentially pseudo-injective module with finite uniform dimension has the internal cancellation property.

Recall that every right $R$-module over a right Noetherian ring $R$ is locally Noetherian.

COROLLARY 3.10. If $M$ is a locally Noetherian pseudo-injective module, then $M=A \oplus B$, where $A$ is a maximal quasi-injective summand, $B$ has no quasi-injective summands, and $A$ and $B$ have no nonzero isomorphic submodules.

COROLlaRY 3.11. A locally Noetherian Dedekind-finite pseudo-injective module has internal cancellation property.

\section{References}

[1] S. Alamelu, 'On quasi-injective modules over Noetherian rings', J. Indian Math. Soc. 39 (1975), 121-130.

[2] H. Q. Dinh, 'A note on pseudo-injective modules', Comm. Algebra 33 (2005), 361-369.

[3] N. V. Dung, D. V. Huynh, P. F. Smith and R. Wisbauer, Extending modules, Pitman Research Notes in Mathematics Ser. 313 (Pitman, 1994).

[4] N. Er, 'Direct sums and summands of weak-CS modules and continuous modules', Rocky Mountain J. Math. 29 (1999), 491-503.

[5] — 'When submodules isomorphic to complements are complements', East-West J. Math. 4 (2002), 1-12.

[6] K. R. Goodearl, Singular torsion and the splitting properties, Amer. Math. Soc. Mem. 124 (Amer. Math. Soc., Providence, RI, 1972).

[7] D. V. Huynh, 'A right countably sigma-CS ring with ACC or DCC on projective principal right ideals is left artinian and QF-3', Trans. Amer. Math. Soc. 347 (1995), 3131-3139.

[8] S. K. Jain and S. Singh, 'On pseudo injective modules and self pseudo injective rings', $J$. Math. Sci. 2 (1967), 23-31.

[9] _ ' 'Quasi-injective and pseudo-injective modules', Canad. Math. Bull. 18 (1975), 359-366.

[10] S. H. Mohamed and B. J. Müller, Continuous and Discrete Modules (Cambridge University Press, Cambridge, 1990).

[11] M. Okado, 'On the decomposition of extending modules', Math. Japonica 29 (1984), 939-941.

[12] B. L. Osofsky, 'Rings all of whose finitely generated modules are injective', Pacific J. Math 14 (1964), 646-650.

[13] S. Singh, 'On pseudo-injective modules', Riv. Mat. Univ. Parma (2) 9 (1968), 59-65.

[14] M. L. Teply, 'Pseudo-injective modules which are not quasi-injective', Proc. Amer. Math. Soc. 49 (1975), 305-310.

[15] A. A. Tuganbaev, 'Pseudo-injective modules and automorphisms', Trudy Sem. Petrovsk. 4 (1978), 241-248. 
[16] L. Zhongkui, 'On X-extending and X-continuous modules', Comm. Algebra 29 (2001), 2407-2418.

Department of Mathematics

Ohio University

Athens, $\mathrm{OH} 45701$

USA

e-mail: noyaner@yahoo.com
Department of Mathematics The Ohio State University-Newark OH 43055

USA 